\title{
La hipnosis en la terapia cognitivo-conductual: Aplicaciones en el campo de las adicciones
}

\author{
Molina del Peral, J.A. \\ Psicólogo Clínico. Práctica privada.
}

\section{RESUMEN}

En este artículo se pretende mostrar cómo la hipnosis puede incorporarse a las intervenciones cognitivo-conductuales, detallándose específicamente cuál es el modelo de trabajo propuesto en el ámbito de las adicciones. Se ha realizado un recorrido por las principales técnicas de intervención de este modelo en adicciones, demostrando cómo las técnicas de sugestión e hipnosis pueden utilizarse como coadyuvantes de las estrategias cognitivo-conductuales. Se concluye que el añadir hipnosis produce un efecto de sinergia en las terapias que se integra, por lo que la utilización de las técnicas sugestivas dentro del campo de las adicciones, puede aumentar la eficacia de estas intervenciones.

Palabras clave: Hipnosis, sugestión, terapia cognitivo-con ductual, adicción.

\begin{abstract}
The goal of this paper is to show in which way hypnosis can be incorporated in cognitive-behavioral therapies. The model of work suggested to follow in addictions field is specifically detailed. The main techniques have been examined and it has been demostrated the usefulness of suggestion techniques as an adjunct of cognitive-behavioral strategies. In conclusion, hypnosids added to therapy causes a synergic effect. Therefore, to use suggestive techniques in addiction field increases the therapy efficacy.
\end{abstract}

Key words: Hypnosis, suggestion, cognitive-behavioral therapy, addiction.

\section{INTRODUCCIÓN}

C uando nos adentramos en el estudio de la hipnosis nos encontramos, si se nos permite la expresión, con un "terreno farragoso". En primer lugar, existe una gran complejidad para dar una definición operativa del término hipnosis que satisfaga a los estudiosos, con lo que no siendo excesivamente exagerados, podríamos decir que nos encontramos tantas definiciones como personas trabajan en este campo. En segundo lugar, hay grandes enfrentamientos entre los defensores de la hipnosis como un estado alterado de conciencia, frente a los que mantienen que no lo es (véase González Ordi, Miguel Tobal y Tortosa, 1992 ; Tortosa, González Ordi y Miguel Tobal, 1993 ; Tortosa, González Ordi y Miguel Tobal, 1999). Este enfrentamiento subyace del tradicional conflicto entre la escuela conductual-cognitiva y la psicoanalítica (Jara, 1994). Por último, los espectáculos televisivos, junto con la literatura, han contribuido de forma notable a fomentar una serie de mitos y concepciones erróneas hacia la hipnosis, que es importante modificar antes de iniciar la intervención (véase Capafons, 1998, González Ordi y Molina, 2000).

Todo lo anteriormente afirmado no facilita el que se conozcan las diferentes posibilidades que ofrece la hipnosis, pero ¿cuáles son éstas en realidad?, ¿es una técnica milagrosa que todo lo "cura"? o por el contrario, algo que es un simple truco. De lo que sí estamos seguros es que ni lo uno, ni lo otro, se acerca a la realidad. En la actualidad, el uso de las técnicas de sugestión e hipnosis debemos entenderlo como un conjunto de técnicas que facilitan el modo de operar 
de otras que han resultado eficaces dentro del campo de la salud mental.

Si hay algo que especialmente se desea destacar es que la hipnosis no es una terapia per se, más bien podría entenderse como un adjunto a la terapia (Lankton, 1982; González Ordi y Miguel Tobal, 1993; Kirsch et al., 1993; Jara y Martínez, 1999; Yapko, 1999). Algunos ejemplos de ello en el campo de las adicciones pueden consultarse en (Cohen, 1984; Manganiello, 1984; Linn, et al., 1993; Molina, 2000; Mendoza, 2000).

A pesar del creciente aumento de publicaciones sobre hipnosis, su aplicación en adicciones no es todavía muy prolijo, limitándose en gran parte a estudios de caso y en un porcentaje muy alto sobre tabaquismo. Por ello, es interesante destacar el trabajo de Manganiello (1984), al no referirse a un estudio de caso, no ser sobre tabaquismo y utilizar la hipnosis junto con otras estrategias. Dicho autor examinó el efecto de la hipnosis como coadyuvante de un programa estándar psicoterapéutico para adictos a la metadona. La muestra estaba compuesta por 70 sujetos de entre 21-63 años que fueron asignados a uno de estos grupos: a) grupo experimental (hipnosis y psicoterapia); b) grupo control (psicoterapia). Después del tratamiento y a los seis meses de seguimiento, los sujetos fueron entrevistados con el objetivo de comparar ambos grupos para determinar si existían diferencias significativas en: número de abstinentes; reducción media de dosis de metadona; menor uso de drogas ilícitas y grado de bienestar. Se encontraron diferencias significativas en todas las medidas en favor del grupo experimental. En consonancia con esto, los diferentes meta-análisis han demostrado que la adición de hipnosis a los tratamientos cognitivoconductuales y psicodinámicos mejora sustancialmente la eficacia de éstos (Smith, Glass y Miller, 1980; Kirsch, Montgomery y Sapirstein, 1995).

Como hemos podido apreciar, la principal utilidad de la hipnosis es como coadyuvante de otras técnicas. Meichenbaun (1988) destaca la importancia de los paquetes combinados de técnicas, en lugar de emplear técnicas aisladas, como medio para afrontar con éxito la intervención clínica, siendo dentro de estos paquetes donde la hipnosis puede mostrarse especialmente eficaz.

\section{OBJETIVOS}

El objetivo de este trabajo es presentar las principales técnicas de intervención en adicciones y como podemos implementarlas en hipnosis. Como observaremos, no hay nada que podamos hacer en hipnosis que no se pueda realizar sin ella, pero destacando lo afirmado anteriormente, la hipnosis produce un efecto sinérgico en las terapias que se integra (Smith et al.,
1980; Kirsch et al., 1995 ; Kirsch et al., 1998), por lo que resulta adecuado, mostrar el modelo de trabajo que podemos utilizar con hipnosis, dentro de las intervenciones cognitivo-conductuales, en el campo de las adicciones.

Lo que haremos es exponer unas líneas de actuación comunes, ya que como ha sido bien referido en la literatura, todas las adicciones presentan muchos aspectos en común. En algunos momentos y para hacer más comprensiva la exposición, intercalaremos algunas sugestiones tipo. Es importante que resaltemos que siempre debemos adaptar las sugestiones a cada persona y no al contrario, por lo que su inclusión es con un objetivo puramente didáctico y no para que se sigan literalmente en las intervenciones, ya que resulta conveniente que los distintos tipos de sugestiones se construyan con la ayuda del paciente.

\section{USO DE LA HIPNOSIS EN ADICCIONES}

Seguidamente, detallaremos las distintas estrategias más comúnmente utilizadas en el ámbito de las intervenciones cognitivo-conductuales en adicciones y como las técnicas de sugestión e hipnosis puede adaptarse a éstas.

\section{TERAPIA DE EXPOSICIÓN}

Existen una serie de estímulos, ya sean externos o internos, que se encuentran fuertemente condicionados al consumo (una hora determinada, celebración de una fiesta, estar sólo y aburrido...). Al tratarse de respuestas condicionadas, pueden aplicarse procedimientos de exposición para extinguirlas, en relación con las señales que las elicitan (Graña, 1994). Con la terapia de exposición se extinguen las respuestas de deseo y los síntomas de abstinencia que se desencadenan ante objetos, pensamientos, lugares, personas, etc. Esto lo podemos hacer primero bajo hipnosis y una vez superado, pasar a una exposición in vivo con prevención de respuesta. El uso de la hipnosis como método de extinción es una de sus aplicaciones más antigua y más ampliamente utilizada (Wolberg, 1948).

La exposición en hipnosis puede ser muy efectiva, ya que ésta favorece la aparición de una serie de fenómenos psicológicos y psicofisiológicos (Miguel Tobal y González Ordi, 1988).

a) Aumento de la capacidad de imaginería mental. Parece que en el proceso de inducción hipnótica, la aparición de sensaciones de relajación y la restricción sensorial, aumentan la capacidad del sujeto para imaginarse intensa y vívidamente escenas sugeridas por el terapeuta o generadas espontáneamente por el propio paciente (Hilgard, 1974; Gibson y Heap, 1991). 
b) Aumento de la implicación emocional respecto a las situaciones imaginadas por el sujeto o sugeridas por el experimentador. Bajo hipnosis, los sujetos tienen la sensación de verse absorbidos por las situaciones o estados emocionales sugeridos por el experimentador, experimentándolos como si fueran reales (Tellegen y Atkinson, 1974). La implicación emocional es uno de los aspectos de la hipnosis que más interés está despertando debido a las implicaciones clínicas que se pueden derivar de ello (González Ordi y Miguel Tobal, 1993).

c) Focalización de la atención en una situación estimular restringida. Las sugestiones verbales dadas por el experimentador, junto con la restricción sensorial y la estimulación repetitiva, llevan al individuo hipnotizado a una progresiva focalización de la atención, y atención selectiva, que provoca que las sugestiones verbales cobren especial relevancia, al ser éstas la fuente principal de información que posee el sujeto en ese momento (Bowers, 1976 ; Wickramasekera, 1988).

Una forma de trabajo sería realizar una jerarquía de señales indicando el deseo estimado que elicitan. En el caso de un fumador (observar a otras personas fumando, ver un cenicero, hablar por teléfono...); con jugadores patológicos (entrar en una sala de juego, escuchar la música de la máquina...). Mientras le exponemos en hipnosis, progresivamente, a estas situaciones, es conveniente dar sugestiones de relajación y autoeficacia "mientras realizas tres respiraciones lentas y profundas, el deseo va descendiendo 6, 5 ... cada vez de menor intensidad, muy bien cada vez más fácil... Estas mismas sugestiones suelen reforzarse al finalizar con el proceso de extinción.

Otra manera de realizar una exposición cognitiva es la sensibilización encubierta (Cautela, 1967). Esta técnica es equivalente al procedimiento operante de castigo positivo y pretende la disminución de la probabilidad de ocurrencia de una conducta. Aquí lo que hacemos es elaborar escenas relacionadas con el consumo (p.e. tomar una cerveza en un bar) y de sensibilización, la que más aparece en la literatura es el vómito. Habitualmente se añaden sensaciones agradables y de alivio al rehusar realizar la conducta no adaptativa. Es conveniente que al paciente se le instruya en autohipnosis para que pueda practicar en casa la conducta a extinguir y la escena de sensibilización, de la misma forma en la que se ha trabajado durante la sesión hipnótica. Cautela (1975) destaca el papel que puede tener la hipnosis como coadyuvante del condicionamiento encubierto.

Una posible forma de instruir a un paciente en autohipnosis es mediante sugestiones posthipnóticas. Estas sugestiones se dan durante la hipnosis, para ser llevadas a cabo cuando nos encontramos fuera de ella. Este método fue desarrollado inicialmente por Salter (1941). Antes de concluir la sesión hipnótica, se le administran al paciente sugestiones poshipnóticas de la siguiente forma:" cada vez que desees trabajar con hipnosis podrás realizarlo de forma sencilla, bastará con que realices varias respiraciones profundas, dejándote llevar, como lo estás haciendo ahora, una vez te sientas a gusto y relajado irás acercando tu mano hacia la frente de forma lenta y progresiva, y así justo en el momento en que la mano toque tu frente te encontrarás en ese estado profundo que ahora tienes, mientras tanto, podrás administrarte todas aquellas sugestiones que hemos trabajado en la consulta".

\section{REESTRUCTURACIÓN COGNITIVA EN HIPNOSIS}

La base de las técnicas de reestructuración cognitiva se encuentra en el pensamiento filosófico de los estoicos, que sostenían que la perturbación emocional no está directamente provocada por la situación, sino por la interpretación que los sujetos hacen de ellas . Las técnicas cognitivas se dirigen a la modificación de las creencias irracionales del paciente. Una de las creencias que más nos describen estos pacientes es "Siempre tendré ganas de consumir". Un ejemplo de como podemos trabajar con ello sería el que a continuación describimos. Una vez realizada la inducción hipnótica le pedimos que "imagine alguna situación que pensara en el momento que le afectaría siempre, p.e. cuando te deja la primera relación... observa el sufrimiento que ahora te genera, así las cosas se modifican con el tiempo, de igual forma los impulsos por el consumo o deseos también cambian, siendo cada vez menos y de menor intensidad, igual que el recordatorio de algo que nos hizo sufrir en un momento dado". Así, le ayudamos a recordar experiencias pasadas en las que tuvo buen resultado. Es importante que tengamos en cuenta las pautas de conducta y habilidades de afrontamiento de su historia personal, las cuales le han resultado eficaces.

Durante la hipnosis podemos plantear una serie de pensamientos alternativos al irracional que presenta el sujeto. Esto se facilita por el hecho de que una de las características de la hipnosis es que el sujeto tiende a seguir literalmente las instrucciones del experimentador, lo que conlleva una aceptación no crítica de estas, debido a que éste se encuentra en una actitud más receptiva (Kroger, 1963; Bowers, 1976; Wolberg, 1982; Gibson y Heap, 1991). Esta actitud se producirá, siempre que hayamos explicado al paciente en que consiste esta técnica y se hayan modificado los principales mitos y concepciones erróneos que la acompañan. De esta forma, será más fácil que se acepte alguno de los pensamientos alternativos, reforzándole el hecho 
de que esta forma de pensar, le hará sufrir menos y le va a ayudar en su recuperación. Golden et al. (1987) destacan diferentes tipos de pensamientos negativos que mantienen la adicción : pensamientos de baja tolerancia a la frustración respecto al abandono, pensamientos autoculpabilizadores por ser adictos y excusas para justificar su adicción.

\section{CONTROL DEL DIÁLOGO INTERNO Y AUTOINSTRUCCIONES}

Kazdin (1983) señala al francés Émile Coué (1927) como uno de los precursores de las estrategias de modificación del diálogo interno. Coué entrenaba a los pacientes para que se dijeran una serie de autoverbalizaciones (autosugestiones) a lo largo del día y en diferentes situaciones. El contenido de las autosugestiones enfatizaban el hecho de que se estaba recuperando, que se sentía cada vez mejor, etc.

Cada uno de nosotros poseemos un "crítico interno" que en diferentes momentos nos manda mensajes. Dichos mensajes pueden, en muchas ocasiones, llevarnos a no hacer cosas que nos apetecen, es decir nos pueden condicionar en nuestra vida cotidiana. El cómo hablamos con nosotros mismos, tiene una gran importancia, pero en el caso de un adicto puede ser vital. Algunos mensajes del "crítico interno" son: "vas a ser un Yonki toda tu vida", "no vas a aguantar sin consumir" "por una cerveza no pasa nada"... Es obvio que estos mensajes regulan nuestra esfera emocional, así hay que aprender a que no nos dominen, existiendo diferentes estrategias para "no creer a esta voz". En hipnosis se puede trabajar, para cada vez que aparezcan estos mensajes, utilizar alguna estrategia para hacerla menos verosímil y sustituir el mensaje por otro más adaptativo.

¿Cómo hacer para no creer al "crítico interno"? El Dr. Michael Yapko durante un seminario de hipnosis clínica y depresión, celebrado en Madrid a principios del año 2000, proponía a los alumnos el que nos autoaplicáramos una serie de mensajes negativos y observáramos como nos sentíamos, al rato y con gran sentido del humor pidió que le diéramos una identidad a este crítico interno que nos hiciera gracia, p.e. algún personaje de dibujos animados y que nos repitiésemos los mismos mensajes, es evidente que lo que producía en los asistentes eran carcajadas. De esta forma, o con cualquier otra forma que se nos ocurra, podemos apreciar como los mismos mensajes, nos afectan emocionalmente de forma diferente.

\section{CONTROL DE LA ANSIEDADY UTILIZACIÓN DE SUGESTIONES POSTHIPNÓTICAS}

La ansiedad se encuentra asociada a distintos trastornos entre los que se encuentra las conductas adictivas (Miguel Tobal, 1996). Un entrenamiento en hipnosis con sugestiones de relajación y visualización de escenas agradables puede servir al sujeto como respuesta de afrontamiento alternativa ante situaciones o emociones negativas y para combatir los síntomas del síndrome de abstinencia.

Esta línea de trabajo fue empleada por Drysdale (1996) con un sujeto de 28 años adicto a la heroína. Dicho autor realizó tres sesiones de hipnosis dentro de un programa más completo para facilitar relajación y visualización de escenas agradables, con un buen resultado en el tratamiento.

Es característico en los adictos manifestar reacciones de ira e impulsividad (Graña, 1994). En hipnosis podemos asociar una palabra clave (p.e. "relax") o escena (p.e. "una playa") a la reducción de los niveles de activación. Lo que haríamos es inducir situaciones de ira, que nos describa lo que siente y posteriormente dar sugestiones para mitigar estas sensaciones. Todo esto lo asociamos a la palabra o escena clave descrita mediante sugestiones posthipnóticas. De esta forma, cada vez que sintiera estas reacciones bastaría con aplicarse esa clave posthipnótica, para adquirir cierto control sobre la situación. Como podemos apreciar, las sugestiones posthipnóticas pueden proporcionar al paciente una adecuada estrategia de autocontrol (González Ordi y Miguel Tobal, 1993). Esto no funciona de forma mágica, sino que requiere de ciertos ensayos para que se instaure y que se siga reforzando para no extinguirse. Mi experiencia y la de otros colegas que trabajan con hipnosis, nos muestra que la principal ventaja de utilizar estas sugestiones posthipnóticas es la rapidez con la que se producen, comparado con un condicionameinto tradicional, es decir, se requiere de un menor número de ensayos. De cualquier forma, necesitamos adecuadas investigaciones para poder soportar lo anteriormente afirmado.

La hipnosis puede ser una estrategia favorecedora de las principales estrategias cognitivo-conductuales mediante sugestiones posthipnóticas (González Ordi y Miguel Tobal, 1993; Vallejo, 1993). Este tipo de sugestiones pueden utilizarse en el contexto del manejo de autoinstrucciones, cambio de pensamiento, puesta en marcha de distintas estrategias de afrontamiento, o para favorecer el autocontrol ante situaciones problema. Un ejemplo podría ser: "cuando se acerque a ese lugar en el que ha consumido habitualmente y sienta como su corazón se acelera, y un gran deseo por consumir, pensará en su palabra clave "calma" y notará como inmediatamente su corazón laterá a un ritmo 
adecuado y usted será el dueño de los actos para así no consumir, de la misma forma en la que lo hemos trabajado durante la hipnosis". Otra forma sería, que las sugestiones poshipnóticas sean un recordatorio para la utilización de otras técnicas "cuando sienta ganas de consumir, recordará que dispone de un amplio número de estrategias, como cambiar de actividad, hacer varias respiraciones profundas, etc.".

\section{EXPECTATIVAS PARA PROMOVER EL CAMBIO}

Uno de los principales objetivos que nos planteamos cuando empezamos a trabajar con un paciente es que se genere una alta motivación para facilitar el cambio. Podemos pensar que el mero hecho de acudir a consulta supone la existencia de esta motivación, lo cual es cierto, pero hay que tener en cuenta que muchos pacientes acuden sometidos a grandes presiones. Esto es especialmente relevante en este campo objeto de estudio, donde se producen situaciones límites "vengo a tratamiento porque mi mujer me ha dicho que si no me abandona, pero a mi no me pasa nada". En un principio suele existir negación de la adicción, lo cual es muy importante se trabaje para conseguir reconocimiento de ésta.

La hipnosis puede favorecer la motivación al cambio, ya que en poco tiempo podemos conseguir ciertas cosas. Con esto no se pretende reforzar la imagen mágica y un tanto esotérica que acompaña a esta técnica. Pongamos algunos ejemplos; ya que una de las características de la hipnosis es la sensación subjetiva de relajación profunda que genera, solemos encontrar que los pacientes nos dicen que la experiencia ha sido muy agradable y especialmente relajante, "una relajación muy profunda", en la línea de lo afirmado por Wolberg (1982) que sugería que la hipnosis provoca un grado de relajación rápido y profundo. Así, bajo hipnosis se reduce el nivel de ansiedad, agitación, características estas muy habituales con las que nos llega una persona adicta. De esta forma y al comprobar como su nivel de activación desciende, el paciente tiene la experiencia de que las cosas se pueden modificar. Otro ejemplo sería cuando realizamos alguna prueba de sugestionabilidad, como la levitación del brazo, o al realizar una anestesia en guante y no le duele al pincharle con una aguja, al haberse modificado la percepción del dolor. Esto a menudo puede convencer al paciente de que la hipnosis puede ayudarles a resolver sus problemas. Lo que nos interesa destacar es como el paciente puede percibir que las cosas pueden modificarse, cambian las expectativas de respuesta (Kirsch, 1990; Jara y Martínez, 1999), lo que en muchos casos supone que el sujeto se implique en el proceso terapéutico.

\section{REPRODUCCIÓN VOLUNTARIA DE LOS EFEC- TOS DE LA SUSTANCIA}

Desde el paradigma del condicionamiento clásico se ha constatado la reproducción de los efectos de las drogas a partir de estímulos inicialmente neutros (Amigó, 1993). Esto ya se conoce desde que Paulov (1927) apareó apomorfina con un tono, realizándose este experimento con un perro de laboratorio. Una de las potencialidades de la hipnosis es poder recrear hipnóticamente las sensaciones positivas de la sustancia sin tomarla realmente (Yapko, 1999). En esta línea, contamos con los clásicos trabajos de Bauman (1971) y Granone (1973), los cuales consiguieron que los sujetos, mediante sugestiones, reprodujeran los efectos de distintas drogas. Esto sin duda es una gran potencialidad, ya que generar los mismos efectos de ciertas drogas, mediante estrategias psicológicas, supone un amplio abanico de implicaciones terapéuticas. Si consideramos el caso de ciertas drogas utilizadas como medicamentos, debemos tener en cuenta los efectos secundarios que producen, así como los fenómenos de tolerancia, dependencia y síndrome de abstinencia que generan. Estos efectos podrían evitarse si reprodujésemos los efectos positivos mediante procedimientos hipnóticos, o por terapia de autorregulación (Amigó, 1992a). Capafons (1993) afirma que la terapia de autorregulación se fundamenta en el uso terapéutico del fenómeno denominado recuerdo sensorial. Se divide en tres fases. En la primera se realizan ejercicios de recuerdo sensorial. En la segunda fase, el sujeto experimenta repetidamente determinadas sensaciones físicas, como respuesta a ciertas imágenes o autosugerencias. A partir de ahí, y ya en la última fase, el paciente está altamente receptivo a cualquier tipo de sugestiones.

En nuestro país ha sido mediante este tipo de terapia donde se han obtenido la reproducción de diferentes drogas, como la cafeína (Amigó, 1992a), anfetamina (Amigó, 1992b), cocaína y éxtasis (Amigó, 1993), efedrina (Amigó, 1994) y heroína (Amigó y Sanchís, 1995; Amigó y Infanzón, 1999).

\section{PREVENCIÓN DE RECAÍDAS EN HIPNOSIS}

En el campo de las adicciones el gran "caballo de batalla" es la elevada tasa de recaídas una vez finalizado el tratamiento. Esto nos hace tratar de implementar nuevas técnicas y contrastar empíricamente su resultado. Una posible estrategia a utilizar es la planificación de situaciones de crisis. Una vez realizada la inducción hipnótica la idea es colocar al paciente en una situación que ha descrito como de alta probabilidad de recaída, haciéndola lo más real posible y utilizar las técnicas de las que dispone, o implementare alguna nueva, para afrontar con éxito esta situación. Es 
importante que resaltemos la utilización de sugestiones de autoeficacia "cada vez te será más sencillo enfrentarte a estas situaciones" durante el proceso y cada vez que afronta alguna situación. Así el sujeto lo que hace es imaginarse haciendo frente a situaciones conflictivas, esto es un modelado encubierto (véase Molina, 2000).

La utilización de estrategias de prevención de recaídas en hipnosis puede ser algo muy novedoso y de gran utilidad, pero a día de hoy, no existe suficiente apoyo empírico, sobre este "prometedor" modelo de trabajo.

\section{CONCLUSIONES}

Todas y cada una de las diferentes técnicas que se han detallado pueden utilizarse en hipnosis, pero también pueden realizarse sin ella, siendo esta la forma tradicional de trabajo. Entonces ¿qué es lo que justifica la inclusión de esta técnica dentro de los paquetes terapéuticos? Una de las grandes ventajas de la utilización de la hipnosis es la gran versatilidad de esta técnica (Karlin y McKeon, 1976), ya que posibilita la combinación de diferentes técnicas en la misma sesión. Así p.e. mientras se expone al paciente a una situación de alta probabilidad de recaída, se puede realizar un modelado encubierto, disminuir la activación asociada a la situación, añadir una reestructuración cognitiva, reforzar ciertas autoinstrucciones... Otra excelencia de la hipnosis es la reducción del tiempo que se emplea para el tratamiento (Dengrove, 1973; Ellis, 1986). Una última ventaja es que los procedimientos hipnosugestivos sirven como catalizadores terapéuticos gracias a las expectativas y creencias en "el poder de la hipnosis", convirtiéndose así en una profecía autocumplida (Stanton, 1979, 1981). De esta forma, aquellos sujetos que esperan un buen resultado terapéutico tienden a obtenerlo (Lazarus, 1973).

Como muestran los distintos estudios meta-analíticos la hipnosis incrementa la eficacia de las terapias donde se integra (Cardeña et al., 1999). Así, aunque la investigación sobre el uso de la hipnosis en adicciones es limitada, por lo que existe la necesidad de llevar a cabo estudios de investigación con un buen control metodológico para evaluar su eficacia, podemos inferir que si aumenta la eficacia de las intervenciones cognitivo-conductuales y éstas son la base de la exposición reseñada durante este artículo, ¿ por qué no van a colaborar en la eficacia del tratamiento de las adicciones?

Es importante destacar que antes de que cualquier terapeuta se plantee el uso de la hipnosis para el tratamiento de las adicciones, es implescindible que tenga un cierto conocimiento de este campo y de las intervenciones más usualmente utilizadas. Esto es aplicable a cualquier otro trastorno, pero es importan- te destacarlo, ya que existen un gran número de "hipnólogos", gente no profesionales que tras recibir un curso de hipnosis se sienten capacitados para "curar" cualquier patología, y que producen gran daño tanto a los pacientes que tratan, como en los profesionales que pretenden hacer un buen uso de esta herramienta terapéutica.

Para finalizar, comentar que sería deseable que tras la lectura de este trabajo, los distintos profesionales que trabajan en el campo de las adicciones, se planteen la inclusión de técnicas sugestivas dentro de sus paquetes terapéuticos.

\section{BIBLIOGRAFÍA}

AMIGÓ, S. (1992a). Terapia de autoregulación y reproducción voluntaria de los efectos de la cafeína: estudio experimental de caso único Informació Pscológica, 49, 46-50.

AMIGÓ, S. (1992b). Manual de terapia de autorregulación. Valencia : Promolibro.

AMIGÓ, S. (1993). Reproducción voluntaria de los efectos de la cocaína y el extasis por medio de la terapia de autorregulación. Informació Psicológica, 53, 17-26.

AMIGÓ, S. (1994). Self-Regulation therapy and the voluntary reproduction of stimulant effects of ephedrine: possible therapeutic applications. Contemporary Hypnosis, 11,108-120.

AMIGÓ, S. Y SANCHIS, M. (1995). Reproducción voluntaria de los efectos de la heroína con terapia de auto-rregulación: un estudio piloto. Comunicación presentada en el Seminario sobre drogodependencias: aspectos sociales, jurídicos y médico asistenciales. Organizado por la European Law Students' Association. Valencia.

AMIGÓ, S. Y INFANZÓN, M.E. (1999). Heroína sin heroína: la sugestión como sustituto de las drogas. Análisis y Modificación de Conducta, 25 (103), 751-781.

BAUMAN, F. (1971). Hypnosis and the adolescent drug abuser. American Journal of Clinical Hypnosis, 13, 17-21.

BOWERS, K.S. ( 1976). Hypnosis for the seriously curious. New York: W.W. Norton.

CAPAFONS, A. (1993). Hipnosis y terapia de autorregulación. En F.J. Labrador, J.A. Cruzado y M. Muñoz (Eds.), Manual de técnicas de modificación y terapia de conducta, (pp. 457-476). Madrid: Pirámide.

CAPAFONS, A. (1998). Hipnosis clínica: una visión cognitivocomportamental. Papeles del Psicólogo, 69, 71-88.

CAUTELA, J.R. (1967). Covert sensitization. Psychological Reports, 43, 405-415.

CAUTELA, J.R. (1975). The use of covert conditioning in hypnotherapy. International Journal of Clinical and Experimental Hypnosis, 23, 15-27.

COHEN, B.B. (1984). A combined approach using meditation-hypnosis and behavioral techniques in the treatment 
of smoking behavior: Case studies of five stressed patients. International Journal of Psychosomatics, 31(1), 33-39.

COUE, E. (1927). The Practice of Autosuggestion, Nueva York: Doubleday.

DENGROVE, E. (1973). The uses of hypnotic in behavior therapy. International Journal of Clinical and Experimental Hypnosis, 21, 13-17.

DRYSDALE, B (1996). The use of hypnosis with an injecting heroin user: Brief clinical description of a single case. Contemporary Hypnosis, 13 (3), 198-201.

ELLIS, A. (1986). Anxiety about ansiety: The use of hypnosis with Rational-Emotive therapy. En E.T. Dowd y J.M. Healy (Eds.), Case studies in hypnotherapy. New York: Guilford Press.

GIBSON, H.B. Y HEAP, M. (1991). Hypnosis in therapy. Hillsdale: Lea.

GOLDEN, W.L., DOWN, E.T. Y FRIEDBERG, F. (1987). Hypnotherapy : a moderm approach. New York : Pergamon Press.

GONZÁLEZ ORDI, H. Y MIGUEL TOBAL, J.J. (1993). Aplicaciones de las técnicas de hipnosis en el ámbito de la modificación cognitivo-conductual. Informació Psicológica, 53, 41-51.

GONZÁLEZ ORDI, H. Y MIGUEL TOBAL, J.J. (1999). Características de la sugestionabilidad y su relación con otras variables psicológicas. Anales de Psicología, 15(1), 5775.

GONZÁLEZ ORDI, H. Y MOLINA, J.A. (2000). Actitudes hacia la hipnosis: estrategias de cambio. Comunicación presentada en el Symposium Nacional sobre Hipnosis y Psicología Clínica, Fuentes, Usos y Abusos de la Hipnosis en Psicología Clínica. Organizado por la Asociación Española de Psicología Conductual (AEPC). Almería.

GONZÁLEZ ORDI, H., MIGUEL TOBAL, J.J. YTORTOSA, F. (1992). ¿Es la hipnosis un estado alterado de conciencia ? Raíces históricas de una controversia. Revista de Historia de la Psicología, 13 (4), 51-74.

GRANONE, F. (1973). Tratado de Hipnosis. Sofrología. Barcelona: Científico-Médica.

GRAÑA, J.L. (1994). Intervención conductual individual en drogodependencias. En J.L. Graña Gómez (Ed.), Conductas adictivas. Teoría, Evaluación y Tratamiento (pp. 143-190). Madrid: Debate.

HILGARD, E.R. (1965). Hipnotic Susceptibility. New York: Harcout, Brace and World.

HILGARD, J.R. (1974). Imaginative involvement: Some characteristics of the highly hypnotizable and the non-hypnotizable. International Journal of Clinical and Experimental Hypnosis, 22, 138-156.

JARA, P. (1994). Hipnoterapia breve en un caso de trastorno obsesivo-compulsivo: deducciones referentes a la hipno- terapia. Análisis y Modificación de Conducta, 20 (70)، 261-273.

JARA, P.Y MARTÍNEZ, F. (1999). Hipnosis, hipnotizabilidad y expectativas de respuesta: una visión crítica. Anales de Psicología, 15(1), 36-56.

KAZDIN, A.E. (1983). Failure of persons respond to the token economy. En E.G. Foa y P.M.G. Emmelkamp (eds.), Failures in Behavior Therapy, Nueva York: Plenum Press.

KARLIN, R.A. Y MCKEON, P. (1976). The use of hypnosis in multimodal therapy. En A.A. Lazarus (Ed.)., Multimodal behavior therapy. New York: Springer Publishing.

KIRSCH, I. (1990). Changing expectations: A key to efective psychoterapy. Pacific Grove, CA: Brooks/ Cole.

KIRSCH, I., CAPAFONS, A., CARDEÑA, E., Y AMIGÓ, S. (1998). Clinical hypnosis and self-regulation therapy : a cognitive-behavioral perspective. Washington, D.C. : American Psychological Association.

KIRSCH, I., LYNN, S.J. Y RHUE J.W. (1993). Introduction a clinical hypnosis (3-22). En J.W. Rhue, S.J. Lynn y I. Kirsch (eds), Handbook of Clinical Hypnosis. Washington, DC: American Psichological Association.

KIRSCH, I., MONTGOMERY, G. Y SAPIRSTEIN, G. (1995). Hypnosis as an adjunt to cognitive-behavioral psychotherapy. A meta-analysis. Journal of Consulting and Clinicaal Psychology, 63, 214-220.

KROGER, W.S. (1963). Clinical and Experimental Hypnosis. Philadelphia: J.B. Lippincott.

LANKTON, S. (1982). The occurrence and use of trance phenomena in nonhypnotic therapies. En J. Zeig (comp.), Ericksonian approaches to hypnosis and psychotherapy, (132-143). Nueva York: Brunner/Mazel.

LAZARUS, A. (1973). "Hypnosis" as a facilitator in behaviour therapy. International Journal of Clinical and Experimental Hypnosis, 21, 25-31.

LYNN, S.J., NEUFELD, V., RHUE, J.W. Y MATORIN, A. (1993). Hypnosis and smoking cessation: a cognitivebehavioral treatment En J.W. Rhue, S.J. Lynn y I. Kirsch (eds), Handbook of Clinical Hypnosis, (555-586). Washington, DC: American Psichological Association.

MARRIOTT, J. A.; BRICE, G. L. (1990). A single session of hypnosis to stop smoking: A clinical survey. Australian Journal of Clinical Hypnotherapy and Hypnosis, 11, 21-28.

MANGANIELLO, A.J. (1984). A comparative study of hypnotherapy and psychotherapy in the treatment of methadone addicts. American Journal of Clinical Hypnosis, 26(4), 273-279.

MEICHENBAUM, D. (1988). Terapias cognitivas-conductuales. En S.J. Lynn y J.P. Garske (Eds.). Psicoterapias Comtemporáneas. Bilbao: Desclée de Brouwer.

MENDOZA, M.E. (2000). La hipnosis como adjunto en el tratamiento del hábito de fumar. Estudio de caso. Psicothema, 12 (3), 330-338. 
MIGUEL TOBAL, J.J. (1996). La ansiedad. Madrid: Aguilar.

MIGUEL TOBAL, J.J. Y GONZÁLEZ ORDI, H. (1988). La analgesia hipnótica: un análisis de las principales aportaciones experimentales y clínicas de la hipnosis al tratamiento psicológico del dolor. Revista Española de Terapia del Comportamiento, 6, 251-270.

MOLINA, J.A. (2000). El empleo de las técnicas de sugestión como coadyuvantes de los programas multicomponentes en el tratamiento del tabaquismo : Estudio de caso único. Comunicación presentada en el Symposium Nacional sobre Hipnosis y Psicología Clínica, Fuentes, Usos y Abusos de la Hipnosis en Psicología Clínica. Organizado por la Asociación Española de Psicología Conductual (AEPC). Almería.

PAULOV, I.P. (1927). Conditioned reflexes. Londres: Oxford University Press.

Salter, A. (1941). Three techniques of autohypnosis. Journal of General Psychology, 24, 423-438.

SMITH, M.L., GLASS, G.V.Y MILLER, T.I. (1980). The benefits of psychotherapy. Baltimore (MD): John Hopkins University Press.

STANTON, H.E. (1979). The hypnotherapeutic placebo. In G.D. Burrows, D.R. Collison y L. Dennerstein (Eds.), Hypnosis. New York: Elsevier/North Holland Biomedical Press.

STANTON, H.E. (1981). Enuresis, homeopathy, and enhancement of placebo effect. American Journal of Clinical Hypnosis, 24, 59-61.
TELLEGEN, A. Y ATKINSON, G. (1974). Openness to absorbency and self-altering experiences "absorpion": A trit relating to hipnotic susceptibility. Journal of Abnormal Psychology, 83, 268-277.

TORTOSA, F., GONZÁLEZ ORDI, H. Y MIGUEL TOBAL, J.J. (1993). Estado alterado de conciencia vs no-estado. Un formato remozado para una controversia clásica. En A. Capafons y S. Amigó, Hipnosis. Terapia de autoregulación e intervención conductual, (9-43). Valencia :Promolibro.

TORTOSA, F., GONZÁLEZ-ORDI, H. Y MIGUEL-TOBAL, J.J. (1999). La hipnosis. Una controversia interminable. Anales de Psicología, 15(1), 3-25.

VALLEJO, M.A. (1993). Técnicas de sugestión e hipnosis. En M.A. VALLEJOY M.A. RUIZ (EDS.). Manual práctico de modificación de conducta, (533-603). Madrid: Fundación Universidad Empresa.

WICKRAMASEKERA, I. E. (1988). Clinical Behavioral medicine. New York: Plenum Press.

WOLBERG, L.R. (1948). Medical Hypnosis. The principies of hypnotherapy. New York: Grune \& Stratton.

WOLBERG, L.R. (1982). Hipnosis: is it for you?. New York: Dembner Books.

YAPKO, M.D. (1999). Lo esencial de la hipnosis. Barcelona: Paidós (Orig. 1995). 